\title{
Red variables in the OGLE-II database: first results for the LMC
}

\author{
L.L. Kiss, T.R. Bedding \\ School of Physics, University of Sydney, NSW 2006, Australia
}

\begin{abstract}
We present a period analysis of more then 23000 red giants in the Large Magellanic Cloud observed by the OGLE-II microlensing project. Periods combined with the single-epoch 2MASS $J H K_{\mathrm{S}}$ magnitudes revealed the complex distributions of stars in the period-luminosity plane. Besides four different sequences corresponding to different modes of pulsation in AGB stars, we also discovered two distinct and wellseparated sequences below the tip of the Red Giant Branch, consisting of almost 10000 short-period and low-amplitude red variable stars. We propose that the majority are likely to be first ascent red giants, showing radial pulsations in the second and third overtone modes.
\end{abstract}

\section{Introduction}

The period-luminosity (PL) relations of long period variables (LPVs) on the AGB are well documented for the Large Magellanic Cloud (LMC). Observations have revealed that not only Mira stars follow a tight infrared PL relation, but also that semi-regular variables pulsating in overtone modes follow distinct and well-defined PL relations (Wood 2000). In recent years there has been strong interest in pulsating red giants, which is reflected in the increasing number of independent analyses of large photometric databases accumulated by the microlensing surveys (e.g. Groenewegen, these proceedings; Ita et al., these proceedings). This interest is due partly to the role of LPVs in extragalactic distance measurements and partly to the intimate relation between pulsation and mass-loss processes on the AGB, which remains a major uncertainty in our understanding of the late stages of stellar evolution.

Recently, Ita et al. (2002) have reported the presence of many red variable stars in the LMC around the tip of the Red Giant Branch (TRGB), which was interpreted as evidence for global oscillations in first ascent red giants. If confirmed, that would imply that pulsations do occur in a less evolved stage than the AGB, which may have a strong impact on the pulsation-driven mass-loss history. A possible way to do that is provided by the ensemble asteroseismology, which deals with global properties of pulsating stars.

Microlensing surveys have stimulated work in this field of research and one of the most extensive datasets was obtained during the second phase of the Optical Gravitational Lensing Experiment (OGLE-II, Udalski et al. 1997). Our paper presents an analysis of red giant variables taken from this database. 


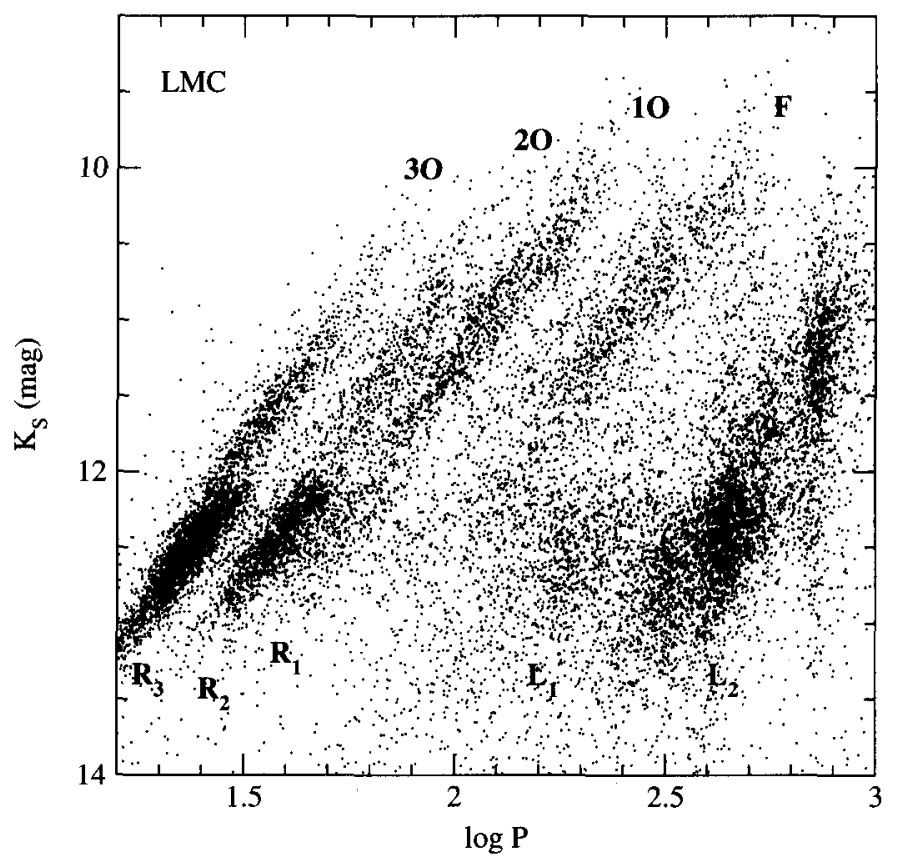

Figure 1. PL relations for red giants in the LMC.

\section{Data analysis}

We have analysed OGLE-II I-band data published by Zebrun et al. (2001). Typical light curves consist of 400 points obtained over four years, so that reliable period determination was possible up to about 500-600 d. We performed the following steps:

1. We made a cross-correlation of the 52937 OGLE-II variables in the LMC with the 2MASS All-Sky Point Source Catalog; with a search-radius of 1 arcsec we found 32062 stars.

2. Red giants were selected by the $J-K_{\mathrm{S}}$ colour; we found 23494 stars with $J-K_{\mathrm{S}}>0.9 \mathrm{mag}$.

3. Four-component light curve fits were calculated by an iterative Fourier analysis (see details in Kiss \& Bedding 2003).

4. We kept only those frequencies larger than $1 / T_{\text {obs }}$ and with semi-amplitudes larger than 5 mmag.

There are some necessary simplifications in this procedure enforced by the huge number of stars. Our sample is more than one order of magnitude larger than that of Wood (2000), so that a number of new features of the PL diagram became visible thanks to the good statistics. 


\section{Results}

We show the resulting PL relations in Fig. 1, where we plot every star just once, using the period of the highest amplitude harmonic component. The figure does not change much when we included all periods for all stars (see Fig. 1, Kiss \& Bedding 2003).

The main features of the diagram are: (i) there is a sudden increase of stellar density below $K_{S}=12.05$, which is located exactly at the TRGB (Cioni et al. 2000); (ii) above the TRGB, there are four sequences identified by Wood $(2000)$ as stars pulsating in fundamental $(\mathrm{F})$, first $(1 \mathrm{O})$, second $(2 \mathrm{O})$ and third (3O) overtone modes (he labelled them as $\mathrm{C}, \mathrm{B}$ and $\mathrm{A}$ ); (iii) below the TRGB, there are two distinct and well-separated sequences $\left(R_{2}\right.$ and $\left.R_{3}\right)$, slightly shifted relative to $2 \mathrm{O}$ and $3 \mathrm{O}$; (iv) there is also a continuation of 10 below the TRGB $\left(R_{1}\right)$, less populated by an order of magnitude than $R_{2}$ and $R_{3}$; and (v) there are two long-period sequences (extending well below the TRGB), one of which has been explained by Wood (2000) as binary variables $\left(\mathrm{L}_{1}=\mathrm{E}\right.$ in Wood 2000) and the other being long secondary periods of ambiguous origin $\left(\mathrm{L}_{2}=\mathrm{D}\right)$. Similar ridges and distributions have also been found for the SMC (Kiss \& Bedding, submitted), which means we did not find strong dependence on metallicity, at least over the range covered by the LMC and SMC.

In order to reveal more information, we plot six different slices of the periodamplitude- $K_{\mathrm{S}}$ magnitude data cube in Fig. 2 , where all periods were taken into account. The amplitudes were calculated as twice the Fourier semi-amplitudes, while we have also indicated three different $J-K_{\mathrm{S}}$ colour ranges in different shades of grey (light grey: $0.9<J-K_{\mathrm{S}} \leq 1.2$; black: $1.2<J-K_{\mathrm{S}} \leq 1.4$; dark grey: $J-K_{\mathrm{S}}>1.4$ ).

There are remarkable features in Fig. 2. Firstly, there is a sudden disappearance of stars below the TRGB with increasing amplitude. For amplitudes larger than $0.04 \mathrm{mag}$, very few short-period stars remain, while for $A>0.14$, almost every star fainter than the TRGB disappears. Secondly, the lower three panels of Fig. 2 show very clearly the fundamental and first overtone sequences and hints of the long secondary period sequence. The upper panels are dominated by the higher overtone pulsators, so that there is a good correlation between the amplitude and mode of pulsation. Thirdly, the colour distributions show the temperature differences within each mode of pulsation very well. The lower three panels are in good agreement with the expectations: for a given mode and assuming a narrow range of masses, the PL relation is equivalent to a densityluminosity relation, which implies a monotonic temperature variation along any particular sequence. $R_{1}, R_{2}$ and $R_{3}$ differ from the other ridges in that each contains both "hot" (light grey) and "warm" (black) red giants along their full extent, with a tendency for warmer stars to have slightly longer periods.

We interpret this last point as evidence for RGB stars mixing with thermally pulsing AGB variables. At the TRGB, RGB and AGB stars have very similar luminosities, with a tendency for RGB stars to have slightly lower temperatures. Recent evolutionary models of the Magellanic Clouds show that for stars with 1$2 \mathrm{M}_{\odot}$, the temperature difference at constant luminosity is $\delta \log T_{\text {eff }} \approx 0.01$. This leads to $\delta \log R \approx-0.02$, or $R_{\mathrm{AGB}} / R_{\mathrm{RGB}} \approx 0.96$. For a given mode of pulsation, the period-density relation $\left(P \sqrt{M / R^{3}}=Q\right)$ results in $\delta \log P=1.5 \times \delta \log R \approx$ 

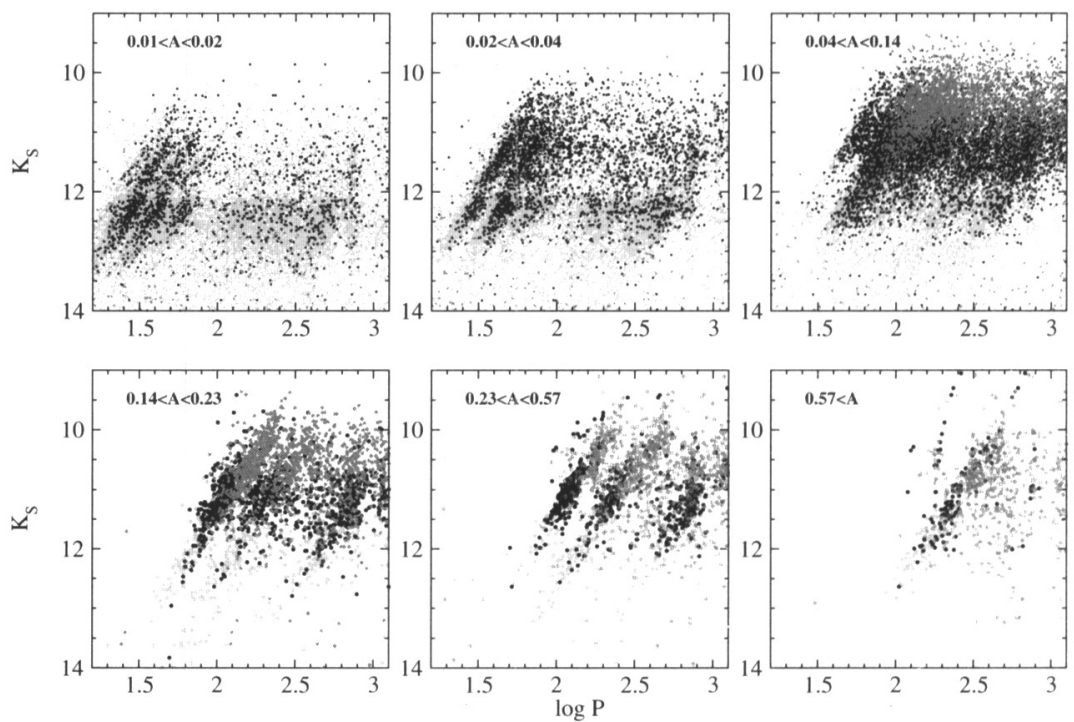

Figure 2. PL relations as function of the full amplitude of modes.

-0.03 , in good agreement with the period shift $\sim-0.05$ within $R_{2}$ and $R_{3}$ in the upper left panel of Fig. 2. On the other hand, the fact that $R_{2}$ and $R_{3}$ are apparent continuations of $2 \mathrm{O}$ and $3 \mathrm{O}$ ridges suggests they are second and third overtone pulsators, a mixture of TPAGB and RGB stars. The apparent displacement of "hot" and "warm" red giants is therefore consistent with the assumption of the same mode and we conclude RGB stars in our sample do pulsate mostly in the second and third overtone modes.

Acknowledgments. This work has been supported by the FKFP Grant 0010/2001, OTKA Grant \#F043203 and the Australian Research Council.

\section{References}

Cioni, M.-R.L., et al. 2000, A\&A, 359, 601

Ita, Y., et al. 2002, MNRAS, 337, L31

Kiss, L.L., Bedding, T.R. 2003, MNRAS, 343, L79

Udalski, A., Kubiak, M., Szymanski, M. 1997, Acta Astron., 47, 319

Wood, P.R. 2000, PASA, 17, 18

Zebrun, K., et al. 2001, Acta Astron., 51, 317 


\section{Discussion}

Christensen-Dalsgaard: A comment: For stochastically excited oscillators the amplitude increases strongly with luminosity (roughly proportionally to $\mathrm{L}$ ); hence it is not surprising that an amplitude-limited sample cuts off at low luminosity. Also, given the identification of stellar oscillators at the base of the red-giant branch ( $\xi \mathrm{Hya}$ ) and at moderate luminosity (as for Arcturus) we would expect to see stochastic oscillators also higher up the RGB and in the AGB. Question: Did you study the statistics of amplitude variations?

Kiss: Yes, we did. The amplitudes change on typical time scales of the few hundred days, which can be also seen in the Fourier-spectra, as broadening of the peaks into groups of peaks under Lorentzian envelopes. Their behaviour is very similar to what is expected for stochastically excited oscillations.

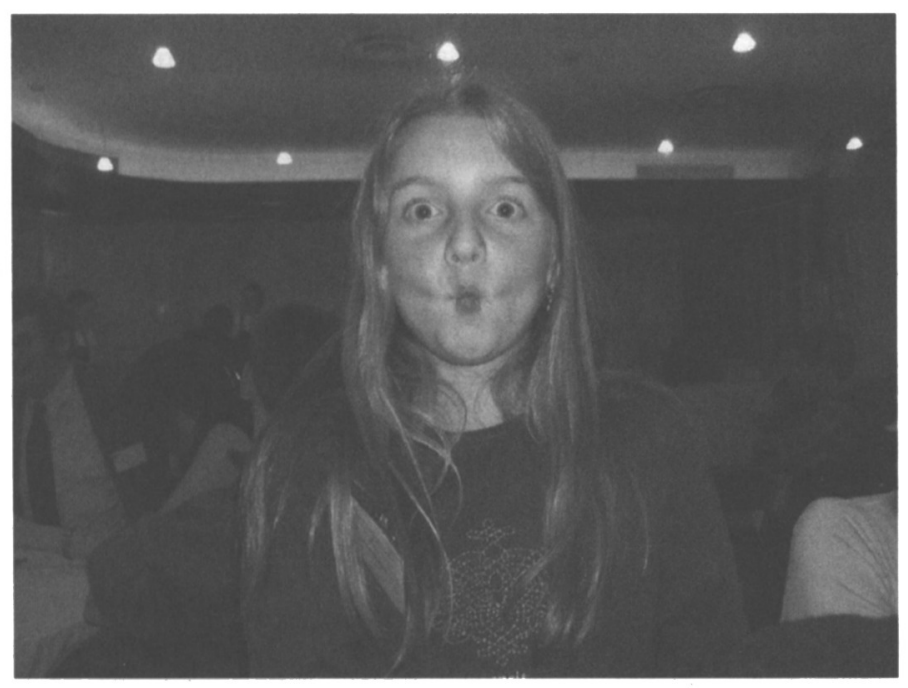

A kiss from An "Little Conny Aerts" Molenberghs 